\title{
NON-TANGENTIAL HALF LIGHTLIKE SUBMANIFOLDS OF AN INDEFINITE COSYMPLECTIC MANIFOLD
}

\author{
DAE HO JIN
}

\begin{abstract}
In this paper, we study half lightlike submanifolds $M$ of an indefinite cosymplectic manifold $\bar{M}$, whose structure vector field is not tangent to $M$. First, we construct two types of such half lightlike submanifolds, named by transversal and normal half lightlike submanifolds. Next, we characterize the lightlike geometries of such two types half lightlike submanifolds.
\end{abstract}

\section{INTRODUCTION}

The geometry of lightlike submanifolds is used in mathematical physics, in particular, in general relativity since lightlike submanifolds produce models of different types of horizons. Lightlike hypersurfaces are also studied in the theory of electromagnetism [5]. As for any semi-Riemannian manifold there is a natural existence of lightlike subspaces, Duggal and Bejancu [5] published their work on the general theory of lightlike submanifolds to fill a gap in the study of submanifolds. Since then there has been very active study on lightlike geometry of submanifolds (see up-to date results in two books $[8,9]$ ). The class of lightlike submanifolds of codimension 2 is composed of two classes by virtue of the rank of its radical distribution, named by half lightlike and coisotropic submanifolds $[4,6,10,11,13]$. Half lightlike submanifold is a special case of $r$-lightlike submanifold $[5,7,14]$ such that $r=1$ and its geometry is more general form than that of coisotrophic submanifold. Much of the works on half lightlike submanifolds will be generalized in a formal way to arbitrary $r$-lightlike submanifolds. Moreover the geometry of half lightlike submanifold is a simple one more than that of the $r$-lightlike submanifold.

Recently several authors studied the geometry of lightlike submanifolds of indefinite cosymplectic manifolds [12] [15]. Most of authors that wrote on lightlike

Received by the editors November 19, 2012. Revised February 7, 2013. Accepted Feb. 22, 2013. 2010 Mathematics Subject Classification. 53C25, 53C40, 53C50.

Key words and phrases. totally umbilical, transversal and normal half lightlike submanifold, indefinite cosymplectic manifold. 
submanifolds $M$ of indefinite almost contact manifolds $\bar{M}$ studied only to the case the structure vector field $\zeta$ of $\bar{M}$ is tangent to $M$, (which are called tangential lightlike submanifold of $\bar{M}$,) but failed to treat with the case $\zeta$ is not tangent to $M$. There are few papers on non-tangential lightlike submanifolds of indefinite almost contact manifold $\bar{M}$ recently studied by Jin $[12,13]$ and Jin-Lee [14].

The objective of this paper is to study the geometry of non-tangential half lightlike submanifolds $M$ of an indefinite cosymplectic manifold $\bar{M}$. There are several different types of half lightlike submanifolds according to the form of the structure vector field of $\bar{M}$. We study two types of them here, named by transversal and normal half lightlike submanifolds. We provide several new results on each types by using the structure of $M$ induced by the structure of $\bar{M}$.

\section{Half Lightlike Submanifolds}

An odd dimensional semi-Riemannian manifold $(\bar{M}, \bar{g})$ is called an indefinite contact metric manifold if there exists an indefinite contact structure $(J, \theta, \zeta, \bar{g})$, where $J$ is a $(1,1)$-type tensor field, $\zeta$ is a vector field which is called the structure vector field of $\bar{M}$ and $\theta$ is a 1-form satisfying

$$
\left\{\begin{array}{l}
J^{2} X=-X+\theta(X) \zeta, J \zeta=0, \theta \circ J=0, \theta(\zeta)=1, \\
\bar{g}(\zeta, \zeta)=1, \bar{g}(J X, J Y)=\bar{g}(X, Y)-\theta(X) \theta(Y), \\
\theta(X)=\bar{g}(\zeta, X), \quad d \theta(X, Y)=\bar{g}(J X, Y)
\end{array}\right.
$$

for any vector fields $X, Y$ on $\bar{M}$. We say that $\bar{M}$ has an indefinite normal contact structure if $N_{J}+d \theta \otimes \zeta=0$, where $N_{J}$ is the Nijenhuis tensor field of $J$. An indefinite normal contact metric manifold is called an indefinite cosymplectic $[12,14,15]$ if

$$
\bar{\nabla}_{X} \theta=0, \quad \bar{\nabla}_{X} J=0,
$$

where $\bar{\nabla}$ is the Levi-Civita connection of $\bar{M}$.

For an indefinite cosymplectic manifold, applying $\bar{\nabla}_{X}$ to $J \zeta=0$ and using the second equation of $(2.2)$ [denote $(2.2)_{2}$ ], we have $J\left(\bar{\nabla}_{X} \zeta\right)=0$. Applying $J$ to this result and using $(2.1)_{1}$ and the fact $\theta\left(\bar{\nabla}_{X} \zeta\right)=0$, we get

$$
\bar{\nabla}_{X} \zeta=0 .
$$

A submanifold $M$ of codimension 2 is called half lightlike submanifold if the rank of the radical distribution $\operatorname{Rad}(T M)=T M \cap T M^{\perp}$ is 1 , where $T M$ and $T M^{\perp}$ are the tangent and normal bundles of $M$ respectively. Then there exists complementary non-degenerate distributions $S(T M)$ and $S\left(T M^{\perp}\right)$ of $\operatorname{Rad}(T M)$ in $T M$ and $T M^{\perp}$ 
respectively, called the screen and co-screen distributions on $M$, such that

$$
T M=\operatorname{Rad}(T M) \oplus_{\text {orth }} S(T M), \quad T M^{\perp}=\operatorname{Rad}(T M) \oplus_{\text {orth }} S\left(T M^{\perp}\right),
$$

where $\oplus_{\text {orth }}$ denotes the orthogonal direct sum. We denote such a half lightlike submanifold by $M=(M, g, S(T M))$. Denote by $F(M)$ the algebra of smooth functions on $M$ and by $\Gamma(E)$ the $F(M)$ module of smooth sections of a vector bundle $E$ over $M$. Choose $L \in \Gamma\left(S\left(T M^{\perp}\right)\right)$ as a unit spacelike vector field without loss of generality. Consider the orthogonal complementary distribution $S(T M)^{\perp}$ to $S(T M)$ in $T \bar{M}$. Certainly $\operatorname{Rad}(T M)$ and $S\left(T M^{\perp}\right)$ are subbundles of $S(T M)^{\perp}$ and

$$
S(T M)^{\perp}=S\left(T M^{\perp}\right) \oplus_{\text {orth }} S\left(T M^{\perp}\right)^{\perp},
$$

where $S\left(T M^{\perp}\right)^{\perp}$ is the orthogonal complementary to $S\left(T M^{\perp}\right)$ in $S(T M)^{\perp}$. It is well-known $[4,6]$ that, for any null section $\xi$ of $\operatorname{Rad}(T M)$, there exists a uniquely defined null vector field $N \in \Gamma(\operatorname{ltr}(T M))$ satisfying

$$
\bar{g}(\xi, N)=1, \quad \bar{g}(N, N)=\bar{g}(N, X)=\bar{g}(N, L)=0, \quad \forall X \in \Gamma(S(T M)) .
$$

Let $\operatorname{tr}(T M)=S\left(T M^{\perp}\right) \oplus_{\text {orth }} l \operatorname{tr}(T M)$. We say that $N, \operatorname{ltr}(T M)$ and $\operatorname{tr}(T M)$ are the lightlike transversal vector field, lightlike transversal vector bundle and transversal vector bundle of $M$ with respect to $S(T M)$ respectively. $T \bar{M}$ is decomposed as

$$
\begin{aligned}
T \bar{M} & =T M \oplus \operatorname{tr}(T M)=\{\operatorname{Rad}(T M) \oplus \operatorname{tr}(T M)\} \oplus_{\text {orth }} S(T M) \\
& =\{\operatorname{Rad}(T M) \oplus l \operatorname{tr}(T M)\} \oplus_{\text {orth }} S(T M) \oplus_{\text {orth }} S\left(T M^{\perp}\right) .
\end{aligned}
$$

Let $P$ be the projection morphism of $T M$ on $S(T M)$. Then the local Gauss and Weingarten formulas for $M$ and $S(T M)$ are given respectively by

$$
\begin{aligned}
& \bar{\nabla}_{X} Y=\nabla_{X} Y+B(X, Y) N+D(X, Y) L, \\
& \bar{\nabla}_{X} N=-A_{N} X+\tau(X) N+\rho(X) L, \\
& \bar{\nabla}_{X} L=-A_{L} X+\phi(X) N \\
& \nabla_{X} P Y=\nabla_{X}^{*} P Y+C(X, P Y) \xi, \\
& \nabla_{X} \xi=-A_{\xi}^{*} X-\tau(X) \xi,
\end{aligned}
$$

for all $X, Y \in \Gamma(T M)$, where $\nabla$ and $\nabla^{*}$ are linear connections on $T M$ and $S(T M)$ respectively, $B$ and $D$ are called the local second fundamental forms of $M, C$ is called the local second fundamental form on $S(T M) . A_{N}, A_{\xi}^{*}$ and $A_{L}$ are linear operators on $T M$, which are called the shape operators, and $\tau, \rho$ and $\phi$ are 1-forms. 
Since $\bar{\nabla}$ is torsion-free, $\nabla$ is also torsion-free, and $B$ and $D$ are symmetric. From the facts $B(X, Y)=\bar{g}\left(\bar{\nabla}_{X} Y, \xi\right)$ and $D(X, Y)=\bar{g}\left(\bar{\nabla}_{X} Y, L\right)$, we know that $B$ and $D$ are independent of the choice of the screen distribution $S(T M)$ and

$$
B(X, \xi)=0, \quad D(X, \xi)=-\phi(X), \forall X \in \Gamma(T M) .
$$

We say that $h(X, Y)=B(X, Y) N+D(X, Y) L$ is the second fundamental form tensor of $M$. The induced connection $\nabla$ of $M$ is not metric and satisfies

$$
\left(\nabla_{X} g\right)(Y, Z)=B(X, Y) \eta(Z)+B(X, Z) \eta(Y),
$$

for all $X, Y, Z \in \Gamma(T M)$, where $\eta$ is a 1 -form on $T M$ such that

$$
\eta(X)=\bar{g}(X, N), \forall X \in \Gamma(T M) .
$$

But the connection $\nabla^{*}$ on $S(T M)$ is metric. The above three local second fundamental forms are related to their shape operators by

$$
\begin{array}{ll}
B(X, Y)=g\left(A_{\xi}^{*} X, Y\right), & \bar{g}\left(A_{\xi}^{*} X, N\right)=0, \\
C(X, P Y)=g\left(A_{N} X, P Y\right), & \bar{g}\left(A_{N} X, N\right)=0, \\
D(X, P Y)=g\left(A_{L} X, P Y\right), & \bar{g}\left(A_{L} X, N\right)=\rho(X), \\
D(X, Y)=g\left(A_{L} X, Y\right)-\phi(X) \eta(Y),
\end{array}
$$

for all $X, Y \in \Gamma(T M)$. By (2.13) and (2.14), we show that $A_{\xi}^{*}$ and $A_{N}$ are $S(T M)$ value shape operators and $A_{\xi}^{*}$ is self-adjoint on $T M$ and

$$
A_{\xi}^{*} \xi=0 \text {. }
$$

Replacing $Y$ by $\xi$ to (2.6) and using (2.10) and (2.11), we have

$$
\bar{\nabla}_{X} \xi=-A_{\xi}^{*} X-\tau(X) \xi-\phi(X) L, \forall X \in \Gamma(T M) .
$$

\section{Transversal Half Lightlike Submanifolds}

Let $M$ be a half lightlike submanifold of an indefinite cosymplectic manifold $\bar{M}$. Due to $[11,12]$, there exists a screen distribution $S(T M)$ such that

$$
J\left(S(T M)^{\perp}\right) \subset S(T M),
$$

which is called a generic screen distribution of $M$. In the sequel, we consider only half lightlike submanifold $M$ equipped with a generic screen distribution and we set $a=\theta(N), b=\theta(\xi)$ and $e=\theta(L)$.

Definition 1. A half lightlike submanifold $M$ of $\bar{M}$ is said to be a transversal half lightlike submanifold [10] if the structure vector field $\zeta$ belongs to $\operatorname{tr}(T M)$. 
For such $M, \zeta$ is expressed as $\zeta=b N+e L$. As $1=\bar{g}(\zeta, \zeta)=e^{2} \bar{g}(L, L)=e^{2}$, we get $e= \pm 1$. Thus we may assume that $e=1$ without loss of generality. Then

$$
\zeta=L+b N
$$

In this section, by saying that transversal half lightlike submanifolds we shall mean half lightlike submanifolds satisfying (3.1) such that $b \neq 0$.

Applying $\bar{\nabla}_{X}$ to $\bar{g}(\zeta, N)=0, b=\bar{g}(\zeta, \xi)$ and the equation (3.1) by turns and using (2.3), (2.7), (2.8), (2.18) and (3.1), we have

$$
\rho(X)=0, \quad X[b]+b \tau(X)=-\phi(X), \quad A_{L} X=-b A_{N} X,
$$

for all $X \in \Gamma(T M)$. Applying $J$ to (3.1) and using the fact $J \zeta=0$, we have $J L=-b J N$. As $J(\operatorname{ltr}(T M))$ and $J\left(S\left(T M^{\perp}\right)\right)$ are distributions of $M$ of rank 1, we see that $J(\operatorname{ltr}(T M))=J\left(S\left(T M^{\perp}\right)\right)$. If $J(\operatorname{Rad}(T M)) \cap J(\operatorname{ltr}(T M)) \neq\{0\}$, then there exists a non-vanishing smooth function $f$ such that $J \xi=f J N$. Then we have $-b^{2}=g(J \xi, J \xi)=f^{2} g(J N, J N)=0$, i.e., $b=0$. It is a contradiction to $b \neq 0$. Thus $J(\operatorname{Rad}(T M)) \cap J(\operatorname{ltr}(T M))=\{0\}$. Consider the local unit timelike vector field $V$ and local unit spacelike vector field $U$ on $S(T M)$ defined by

$$
V=-b^{-1} J \xi, \quad U=-b^{-1}\left\{J \xi+b^{2} J N\right\} .
$$

Then $\{V, U\}$ is an orthonormal frame field of $J(\operatorname{Rad}(T M)) \oplus J(l \operatorname{tr}(T M))$. We know that $J(\operatorname{Rad}(T M)) \oplus J(\operatorname{ltr}(T M))=J(\operatorname{Rad}(T M)) \oplus_{\text {orth }} H_{U}$, where $H_{U}=\operatorname{Span}\{U\}$. From these results and the decomposition $(2.4)_{1}, T M$ is decomposed as follow

$$
T M=\operatorname{Rad}(T M) \oplus_{\text {orth }}\left\{J(\operatorname{Rad}(T M)) \oplus_{\text {orth }} H_{U} \oplus_{\text {orth }} H\right\},
$$

where $H$ is a non-degenerate and almost complex distribution on $M$ with respect to the structure tensor $J$, otherwise $S(T M)$ is degenerate. Applying to (3.3), we get

$$
J V=b^{-1} \xi-b N-L, \quad J U=b^{-1} \xi-L .
$$

These two vector fields are unit timelike and unit spacelike vector fields respectively such that $\bar{g}(J U, J V)=1$. From (3.3) and (3.5) we show that

$$
b N=J U-J V, \quad b J N=V-U=-J L .
$$

Denote by $S$ the projection morphism of $T M$ on $H$. Using (3.4), any vector field $X$ on $M$ is expressed as

$$
X=S X+\eta(X) \xi+v(X) V+u(X) U
$$


where $u, v$ and $\omega$ are 1-forms locally defined on $M$ by

$$
v(X)=-g(X, V), u(X)=g(X, U), \omega(X)=v(X)+u(X) .
$$

Using (3.5), the action $J X$ of $X$ by $J$ is expressed as

$$
J X=F X+b^{-1} \omega(X) \xi-b \eta(X) V-b v(X) N-\omega(X) L,
$$

where $F$ is a tensor field of type $(1,1)$ defined on $M$ by

$$
F X=J S X, \quad \forall X \in \Gamma(T M) .
$$

Applying $\bar{\nabla}_{X}$ to $b V=-J \xi$ and $U=V-b J N$ by turns and using (2.2), (2.18), (3.2), (3.6) and (3.8), we have

$$
\begin{aligned}
& b \nabla_{X} V=F\left(A_{\xi}^{*} X\right)-D(X, V) \xi+\phi(X) U, \\
& b \nabla_{X} U=F\left(A_{\xi}^{*} X+b^{2} A_{N} X\right)-D(X, U) \xi+\phi(X) V, \\
& b D(X, V)=B(X, V-U)=-\omega\left(A_{\xi}^{*} X\right), \\
& b D(X, U)=-\omega\left(A_{\xi}^{*} X+b^{2} A_{N} X\right), \quad \forall X \in \Gamma(T M) .
\end{aligned}
$$

Definition 2. A half lightlike submanifold $M$ of $\bar{M}$ is called totally umbilical[5] if there is a smooth vector field $\mathcal{H}$ on $\operatorname{tr}(T M)$ such that

$$
h(X, Y)=\mathcal{H} g(X, Y), \forall X, Y \in \Gamma(T M) .
$$

In case $\mathcal{H}=0$, i.e., $h=0$ on $\mathcal{U}$, we say that $M$ is totally geodesic.

It is easy to see that $M$ is totally umbilical if and only if there exist smooth functions $\beta$ and $\delta$ on each coordinate neighborhood $\mathcal{U}$ such that

$$
B(X, Y)=\beta g(X, Y), \quad D(X, Y)=\delta g(X, Y), \quad \forall X, Y \in \Gamma(T M) .
$$

Definition 3. A screen distribution $S(T M)$ is called totally umbilical [5] in $M$ if there is a smooth function $\gamma$ on any coordinate neighborhood $\mathcal{U}$ in $M$ such that

$$
C(X, P Y)=\gamma g(X, Y), \forall X, Y \in \Gamma(T M) .
$$

In case $\gamma=0$ on $\mathcal{U}$, we say that $S(T M)$ is totally geodesic in $M$.

Theorem 3.1. Let $M$ be a totally umbilical transversal half lightlike submanifold of an indefinite cosymplectic manifold $\bar{M}$. Then $M$ and $S(T M)$ are totally geodesic.

Proof. If $M$ is totally umbilical, then, from (3.11) and (3.13), we have

$$
b \delta g(X, V)=\beta g(X, V-U), \quad \forall X \in \Gamma(T M) .
$$


Replacing $X$ by $U$ to this, we get $\beta=0$ and then, replacing $X$ by $V$, we have $\delta=0$ as $b \neq 0$. Thus $M$ is totally geodesic. As $D=0$ and $\rho=\phi=0$, we have $A_{L}=0$ by (2.15). Therefore $C=0$ by $(3.2)_{3}$. Thus $S(T M)$ is also totally geodesic in $M$.

Theorem 3.2. Let $M$ be a transversal half lightlike submanifold of an indefinite cosymplectic manifold $\bar{M} . H$ is integrable if and only if

$$
h(X, F Y)=h(F X, Y), \quad \forall X, Y \in \Gamma(H) .
$$

Moreover, if $M$ is totally umbilical, then $H$ is a parallel distribution.

Proof. Taking $Y \in \Gamma(H)$, we get $F Y=J Y \in \Gamma(H)$ by (3.8). Applying $\bar{\nabla}_{X}$ to this and using $(2.2),(2.6),(3.2)_{3},(3.6),(3.7)$ and (3.8), we have

$$
\begin{aligned}
& B(X, F Y)=b g\left(\nabla_{X} Y, V\right), D(X, F Y)=g\left(\nabla_{X} Y, V-U\right), \\
& b\left(\nabla_{X} F\right) Y=-D(X, F Y) \xi-\{B(X, Y)-b D(X, Y)\} U+B(X, Y) V,
\end{aligned}
$$

for any $X \in \Gamma(T M)$ and $Y \in \Gamma(H)$. From two equations of (3.14), we have

$$
h(X, F Y)-h(F X, Y)=b g([X, Y], V) N+g([X, Y], V-U) L .
$$

If $H$ is integrable, then, for any $X, Y \in \Gamma(H),[X, Y] \in \Gamma(H)$. Thus $g([X, Y], V)=0$ and $g([X, Y], V-U)=0$. Consequently $h(X, F Y)=h(F X, Y)$. Conversely if $h(X, F Y)=h(F X, Y)$ for all $X, Y \in \Gamma(H)$, then we have $g([X, Y], V)=0$ and $g([X, Y], U)=0$. These imply $[X, Y] \in \Gamma(H)$. Thus $H$ is integrable.

If $M$ is totally umbilical, then, from Theorem 3.1 and (3.14), we have

$$
g\left(\nabla_{X} Y, V\right)=g\left(\nabla_{X} Y, U\right)=0, \forall X \in \Gamma(T M), \forall Y \in \Gamma(H) .
$$

This imply $\nabla_{X} Y \in \Gamma(H)$ for all $X \in \Gamma(T M)$ and $Y \in \Gamma(H)$. Thus $H$ is parallel.

Theorem 3.3. Let $M$ be a transversal half lightlike submanifold of an indefinite cosymplectic manifold $\bar{M}$. Then $F$ is parallel on $H$ with respect to the induced connection $\nabla$ on $M$ if and only if $H$ is a parallel distribution on $M$.

Proof. Assume that $F$ is parallel on $H$ with respect to $\nabla$. For any $X, Y \in \Gamma(H)$, we have $\left(\nabla_{X} F\right) Y=0$. Taking the scalar product with $V$ and $U$ to (3.15) with $\left(\nabla_{X} F\right) Y=0$, we have $B(X, Y)=0$ and $D(X, Y)=0$ for all $X, Y \in \Gamma(H)$ respectively. From (3.14), we have $g\left(\nabla_{X} Y, V\right)=0$ and $g\left(\nabla_{X} Y, U\right)=0$. These imply $\nabla_{X} Y \in \Gamma(H)$ for all $X, Y \in \Gamma(H)$. Thus $H$ is parallel.

Conversely if $H$ is parallel, from (3.14) we have

$$
B(X, F Y)=0, D(X, F Y)=0, \forall X, Y \in \Gamma(H) .
$$


For any $Y \in \Gamma(H)$, we get $F^{2} Y=J^{2} Y=-Y$. Replacing $Y$ by $F Y$ to (3.16), we have $B(X, Y)=0$ and $D(X, Y)=0$ for any $X, Y \in \Gamma(H)$. Thus $F$ is parallel on $H$ with respect to $\nabla$ by (3.15).

Theorem 3.4. Let $M$ be a transversal half lightlike submanifold of an indefinite cosymplectic manifold $\bar{M}$. If $M$ is totally umbilical, then $M$ is locally a product manifold $M=\mathcal{C}_{\xi} \times \mathcal{C}_{V} \times \mathcal{C}_{U} \times M^{\sharp}$, where $\mathcal{C}_{\xi}, \mathcal{C}_{V}$ and $\mathcal{C}_{U}$ are null, timelike and spacelike curves tangent to $\operatorname{Rad}(T M), J(\operatorname{Rad}(T M))$ and $H_{U}$ respectively and $M^{\sharp}$ is a leaf of the integrable distribution $H$.

Proof. As $M$ is totally umbilical, $H$ is parallel by Theorem 3.2. From (2.10), (3.2) $)_{3}$, (3.9) and (3.10), we have $\nabla_{X} \xi=-\tau(X) \xi$ and $\nabla_{X} V=\nabla_{X} U=0$. Thus $\operatorname{Rad}(T M)$, $J(\operatorname{Rad}(T M))$ and $H_{U}$ are also parallel. Thus we have $M=\mathcal{C}_{\xi} \times \mathcal{C}_{V} \times \mathcal{C}_{U} \times M^{\sharp}$, where $\mathcal{C}_{\xi}, \mathcal{C}_{V}$ and $\mathcal{C}_{U}$ are null, timelike and spacelike curves tangent to $\operatorname{Rad}(\operatorname{TM})$, $J(\operatorname{Rad}(T M))$ and $H_{U}$ respectively and $M^{\sharp}$ is a leaf of $H$.

Definition 4. A half lightlike submanifold $M$ of a semi-Riemannian manifold $\bar{M}$ is said to be irrotational [16] if $\bar{\nabla}_{X} \xi \in \Gamma(T M)$ for any $X \in \Gamma(T M)$.

From (2.18) we show that a necessary and sufficient condition for $M$ to be irrotational is $D(X, \xi)=0=\phi(X)$ for all $X \in \Gamma(T M)$.

Theorem 3.5. Let $M$ be a transversal half lightlike submanifold of an indefinite cosymplectic manifold $\bar{M}$. Then the following are equivalent:

(1) If $V$ is parallel with respect to $\nabla$, then $M$ is irrotational and

$$
D(X, V)=0, \quad A_{\xi}^{*} X=-b B(X, V) J N, \quad \forall X \in \Gamma(T M) .
$$

(2) If $U$ is parallel with respect to $\nabla$, then $M$ is irrotational and

$$
D(X, U)=0, A_{\xi}^{*} X+b^{2} A_{N} X=-b B(X, U) J N, \forall X \in \Gamma(T M) .
$$

Moreover, if $V$ and $U$ are parallel with respect to $\nabla$, then $A_{L}=0$ and $S(T M)$ is totally geodesic in $M$.

Proof. If $V$ is parallel with respect to $\nabla$, then, taking the scalar product with $N$ and $U$ to (3.9) by turns, we have $D(X, V)=0$ and $\phi=0$ ( $M$ is irrotational) respectively. Thus we have $F\left(A_{\xi}^{*} X\right)=0$ for all $X \in \Gamma(T M)$. Using this, (3.8) and (3.11), we get $J\left(A_{\xi}^{*} X\right)=-b v\left(A_{\xi}^{*} X\right) N$. Applying $J$ to this and using $\theta\left(A_{\xi}^{*} X\right)=0$, we obtain (1). In a similar way, if $U$ is parallel with respect to $\nabla$, then, using $(3.2)_{3},(3.7)_{3},(3.8)$, (3.10) and (3.12), we have (2). 
Assume that $V$ and $U$ are parallel with respect to $\nabla$. Substituting $A_{\xi}^{*} X=$ $-b B(X, V) J N$ into $A_{\xi}^{*} X+b^{2} A_{N} X=-b B(X, U) J N$ and using (3.11) and the fact $D(X, V)=0$, we have $A_{N} X=D(X, V) J N=0$. Thus $S(T M)$ is totally geodesic in $M$. By $(3.2)_{3}$ we show that $A_{L}=0$.

Theorem 3.6. Let $M$ be a transversal half lightlike submanifold of an indefinite cosymplectic manifold $\bar{M}$. If $V$ and $U$ are parallel with respect to $\nabla$, then $M$ is locally a product manifold $M=\mathcal{C}_{\xi} \times \mathcal{C}_{V} \times \mathcal{C}_{U} \times M^{\sharp}$, where $\mathcal{C}_{\xi}, \mathcal{C}_{V}$ and $\mathcal{C}_{U}$ are null, timelike and spacelike curves tangent to $\operatorname{Rad}(T M), J(\operatorname{Rad}(T M))$ and $H_{U}$ respectively and $M^{\sharp}$ is a leaf of the integrable distribution $H$.

Proof. As $V$ is parallel with respect to $\nabla$, for any $Y \in \Gamma(H)$ we have

$$
B(X, Y)=g\left(A_{\xi}^{*} X, Y\right)=-b B(X, V) g(Y, J N)=0, \quad \forall X \in \Gamma(T M),
$$

due to (1) of Theorem 3.5. If $U$ is also parallel with respect to the connection $\nabla$, then, since $A_{L}=0$ due to Theorem 3.5, we have

$$
D(X, Y)=g\left(A_{L} X, Y\right)=0, \quad \forall X, Y \in \Gamma(T M) .
$$

From these two results and (3.15), we show that $F$ is parallel on $H$ with respect to the connection $\nabla$. By Theorem 3.3, $H$ is a parallel distribution on $M$. As $V$ and $U$ are parallel with respect to $\nabla$ and $\nabla_{\xi} \xi=-\tau(\xi) \xi$, we have our theorem.

\section{Normal Half Lightlike Submanifolds}

Definition 5. A half lightlike submanifold $M$ of $\bar{M}$ is said to be a normal half lightlike submanifold if the structure vector field $\zeta$ of $\bar{M}$ is normal to $M$.

For such $M, \zeta$ is expressed as $\zeta=a \xi+e L$. As $1=\bar{g}(\zeta, \zeta)=e^{2} \bar{g}(L, L)=e^{2}$, we have $e= \pm 1$. Thus we shall assume that $e=1$ without loss of generality. Then

$$
\zeta=L+a \xi .
$$

In this section, by saying that normal half lightlike submanifold we shall mean half lightlike submanifold satisfying (4.1) such that $a \neq 0$.

Theorem 4.1. Let $M$ be a normal half lightlike submanifold of an indefinite cosymplectic manifold $\bar{M}$. Then $M$ is irrotational.

Proof. Applying $\bar{\nabla}_{X}$ to $\bar{g}(\zeta, \xi)=0$ and using (2.3) and (2.18), we have

$$
\phi(X)=0, \quad \forall X \in \Gamma(T M) .
$$

By Definition 4, it follows from (4.2) that $M$ is irrotational. 
Applying $\bar{\nabla}_{X}$ to $a=\bar{g}(\zeta, N)$ and the equation (4.1) by turns, for any $X \in \Gamma(T M)$, and using (2.3), (2.7) (2.8), (2.18), (4.1) and (4.2), we have

$$
X[a]-a \tau(X)=\rho(X), \quad A_{L} X=-a A_{\xi}^{*} X+\rho(X) \xi .
$$

Taking the scalar product with $Y$ to $(4.3)_{2}$ and using (2.13) and (2.16), we have

$$
D(X, Y)=-a B(X, Y), \quad \forall X, Y \in \Gamma(T M) .
$$

Applying $J$ to (4.1) and using the fact $J \zeta=0$, we obtain $J L=-a J \xi$. Thus we show that $J(\operatorname{Rad}(T M))=J\left(S\left(T M^{\perp}\right)\right)$ and $J(\operatorname{Rad}(T M)) \cap J(\operatorname{ltr}(T M))=\{0\}$.

Consider two local null vector fields $V^{*}$ and $U^{*}$ on $S(T M)$ defined by

$$
V^{*}=-J \xi, \quad U^{*}=-J N-\frac{a^{2}}{2} J \xi .
$$

Then $\left\{V^{*}, U^{*}\right\}$ is a null frame field of $J(\operatorname{Rad}(T M)) \oplus J(\operatorname{ltr}(T M))$ and

$$
J(\operatorname{Rad}(T M)) \oplus J(l t r(T M))=J(\operatorname{Rad}(T M)) \oplus H_{U^{*}},
$$

where $H_{U^{*}}=\operatorname{Span}\left\{U^{*}\right\}$. Thus the decomposition (2.4) is reduced to

$$
T M=\operatorname{Rad}(T M) \oplus_{\text {orth }}\left\{J(\operatorname{Rad}(T M)) \oplus H_{U^{*}} \oplus_{\text {orth }} H^{*}\right\} .
$$

where $H^{*}$ is a non-degenerate almost complex distribution on $M$ with respect to $J$, otherwise $S(T M)$ is degenerate. In this case,

$$
J V^{*}=\xi, \quad J U^{*}=N-a L-\frac{a^{2}}{2} \xi
$$

are null vector fields such that $\bar{g}\left(J U^{*}, J V^{*}\right)=1$. Denote by $S^{*}$ the projection morphism of $T M$ on $H^{*}$. By using (4.6), $J X$ is expressed as follows

$$
J X=\psi X+\omega^{*}(X) \xi-\eta(X) V^{*}+u^{*}(X) N-a u^{*}(X) L,
$$

for all $X \in \Gamma(T M)$, where $u^{*}, v^{*}$ and $\omega^{*}$ are 1-forms locally defined on $M$ by

$$
u^{*}(X)=g\left(X, V^{*}\right), \quad v^{*}(X)=g\left(X, U^{*}\right), \quad \omega^{*}(X)=v^{*}(X)-\frac{a^{2}}{2} u^{*}(X)
$$

and $\psi$ is a $(1,1)$ type tensor field defined by

$$
\psi X=J S^{*} X, \quad \forall X \in \Gamma(T M) .
$$


Applying $\bar{\nabla}_{X}$ to (4.5) and using (2.2), (2.6), (2.7), (2.18), (4.2), (4.3), (4.5), (4.7) and the fact $J L=a V^{*}$, we obtain

$$
\begin{aligned}
& \nabla_{X} V^{*}=\psi\left(A_{\xi}^{*} X\right)+\omega^{*}\left(A_{\xi}^{*} X\right) \xi-\tau(X) V^{*}, \\
& \nabla_{X} U^{*}=\psi\left(A_{N} X+\frac{a^{2}}{2} A_{\xi}^{*} X\right)+\omega^{*}\left(A_{N} X+\frac{a^{2}}{2} A_{\xi}^{*} X\right) \xi+\tau(X) U^{*}, \\
& B\left(X, U^{*}\right)=C\left(X, V^{*}\right)+\frac{a^{2}}{2} B\left(X, V^{*}\right), \quad \forall X \in \Gamma(T M) .
\end{aligned}
$$

Theorem 4.2. Let $M$ be a normal half lightlike submanifold of an indefinite cosymplectic manifold $\bar{M}$. If $M$ and $S(T M)$ are totally umbilical, then $M$ and $S(T M)$ are totally geodesic.

Proof. If $M$ and $S(T M)$ are totally umbilical, from (4.11) we have

$$
\beta g\left(X, U^{*}\right)=\gamma g\left(X, V^{*}\right)+\frac{a^{2}}{2} \beta g\left(X, V^{*}\right), \quad \forall X \in \Gamma(T M) .
$$

Replacing $X$ by $V^{*}$ to this and using the fact $g\left(V^{*}, U^{*}\right)=1$, we have $\beta=0$ and $\gamma g\left(X, V^{*}\right)=0$ for all $X \in \Gamma(T M)$. Replacing $X$ by $U^{*}$ to the last equation, we have $\gamma=0$. Thus $S(T M)$ is totally geodesic in $M$. As $B=0$, we have $D=0$ by (4.4). Thus $M$ is also totally geodesic.

Theorem 4.3. Let $M$ be a normal half lightlike submanifold of an indefinite cosymplectic manifold $\bar{M}$. If $M$ and $S(T M)$ are totally umbilical, then $M$ is locally a product manifold $M=\mathcal{C}_{\xi} \times \mathcal{C}_{V^{*}} \times \mathcal{C}_{U^{*}} \times M^{*}$, where $\mathcal{C}_{\xi}, \mathcal{C}_{V^{*}}$ and $\mathcal{C}_{U^{*}}$ are null, timelike and spacelike curves tangent to $\operatorname{Rad}(T M), J(\operatorname{Rad}(T M))$ and $H_{U^{*}}$ respectively and $M^{*}$ is a leaf of the integrable distribution $H^{*}$.

Proof. Taking $Y \in \Gamma\left(H^{*}\right)$, we have $g\left(Y, V^{*}\right)=0$ and $g\left(Y, U^{*}\right)=0$. Applying $\nabla_{X}$ to these two equations and using (4.7), (4.9) and (4.10), we have

$$
g\left(\nabla_{X} Y, V^{*}\right)=B(X, \psi Y), \quad g\left(\nabla_{X} Y, U^{*}\right)=C(X, \psi Y)+\frac{a^{2}}{2} B(X, \psi Y) .
$$

If $M$ and $S(T M)$ are totally umbilical, then $B=C=0$ by Theorem 4.2. Thus we get $g\left(\nabla_{X} Y, V^{*}\right)=g\left(\nabla_{X} Y, U^{*}\right)=0$. Since $C=0$, we obtain $\nabla_{X} Y=\nabla_{X}^{*} Y$ for all $X \in \Gamma(T M)$ and $Y \in \Gamma\left(H^{*}\right)$. This implies $g\left(\nabla_{X} Y, N\right)=0$. From this result, we see that $H^{*}$ is parallel. As $A_{\xi}^{*}=A_{N}=0$, from $(2.10)$, (4.9) and (4.10), we have

$$
\nabla_{X} \xi=-\tau(X) \xi, \nabla_{X} V^{*}=-\tau(X) V^{*}, \nabla_{X} U^{*}=\tau(X) U^{*},
$$

for all $X \in \Gamma(T M)$. Thus the distributions $\operatorname{Rad}(T M), J(\operatorname{Rad}(T M))$ and $H_{U^{*}}$ are also parallel on $M$. Therefore we have our assertion. 
Taking $Y \in \Gamma\left(H^{*}\right)$, we have $\psi Y=J Y \in \Gamma\left(H^{*}\right)$. Applying $\bar{\nabla}_{X}$ to $J Y=\psi Y$ and using (2.2), (2.6), (4.4), (4.5) and (4.7), we have

$$
\left(\nabla_{X} \psi\right) Y=\omega^{*}\left(\nabla_{X} Y\right) \xi-\left\{C(X, Y)+\frac{a^{2}}{2} B(X, Y)\right\} V^{*}-B(X, Y) U^{*},
$$

for all $X, Y \in \Gamma(T M)$. By the same procedure as for Theorem 3.4 and by using (4.12) and (4.13) instead of (3.14) and (3.15), the following theorem holds:

Theorem 4.4. Let $M$ be a normal half lightlike submanifold of an indefinite cosymplectic manifold $\bar{M}$. Then $\psi$ is parallel on $H^{*}$ with respect to the connection $\nabla$ if and only if $H^{*}$ is a parallel distribution on $M$.

By straightforward calculations from (4.12) and (4.13) and the same method as Theorem 3.5, the following theorem holds:

Theorem 4.5. Let $M$ be a normal half lightlike submanifold of an indefinite cosymplectic manifold $\bar{M}$. Then the following are equivalent:

(1) If $V^{*}$ is parallel with respect to $\nabla$, then we have

$$
A_{\xi}^{*} X=B\left(X, U^{*}\right) V^{*}+B\left(X, V^{*}\right) U^{*}, C\left(X, V^{*}\right)=0, \tau(X)=0 .
$$

(2) If $U^{*}$ is parallel with respect to $\nabla$, then we have

$$
\begin{aligned}
& A_{N} X+\frac{a^{2}}{2} A_{\xi}^{*} X=\frac{a^{2}}{2} B\left(X, U^{*}\right) V^{*}+\left\{\frac{a^{2}}{2} B\left(X, V^{*}\right)+C\left(X, V^{*}\right)\right\} U^{*}, \\
& C\left(X, U^{*}\right)=0, \quad \tau(X)=0, \quad \forall X \in \Gamma(T M) .
\end{aligned}
$$

Moreover, if $V^{*}$ and $U^{*}$ are parallel with respect to $\nabla$, then $S(T M)$ is totally geodesic in $M$. In this case, if $M$ is totally umbilical, then $M$ is totally geodesic.

From Theorem 4.3 and 4.5, we have the following theorem:

Theorem 4.6. Let $M$ be a totally umbilical normal half lightlike submanifold of an indefinite cosymplectic manifold $\bar{M}$. If $V^{*}$ and $U^{*}$ are parallel with respect to $\nabla$, then $M$ is locally a product manifold $\mathcal{C}_{\xi} \times \mathcal{C}_{V^{*}} \times \mathcal{C}_{U^{*}} \times M^{*}$, where $\mathcal{C}_{\xi}, \mathcal{C}_{V^{*}}$ and $\mathcal{C}_{U^{*}}$ are null, timelike and spacelike curves tangent to $\operatorname{Rad}(T M), J(\operatorname{Rad}(T M))$ and $H_{U^{*}}$ respectively and $M^{*}$ is a leaf of the integrable distribution $H^{*}$.

\section{REFERENCES}

1. Călin, C.: Contributions to geometry of CR-submanifold. Thesis, University of Iasi, Iasi, Romania, 1998. 
2. De Rham, G.: Sur la réductibilité d'un espace de Riemannian. Comm. Math. Helv. 26 (1952), 328-344.

3. Duggal, K.L.: On canonical screen for lightlike Submanifolds of codimension 2. Central Europen J. Math. 5 (2007), no. 4, 710-719.

4. Duggal, K.L. \& Bejancu, A.: Lightlike submanifolds of codimension 2. Math. J. Toyama Univ. 15 (1992), 59-82.

5. ___ Lightlike Submanifolds of Semi-Riemannian Manifolds and Applications. Kluwer Acad. Publishers, Dordrecht, 1996.

6. Duggal, K.L. \& Jin, D.H.: Half lightlike submanifolds of codimension 2. Math. J. Toyama Univ. 22 (1999), 121-161.

7. ___ Totally umbilical submanifolds. Kodai Math. J. 26 (2003), no. 1, 49-68.

8. __ N Null curves and Hypersurfaces of Semi-Riemannian Manifolds. World Scientific, 2007.

9. Duggal, K.L. \& Sahin, B.: Differential geometry of lightlike submanifolds. Frontiers in Mathematics, Birkhäuser, 2010.

10. Jin, D.H.: Transversal half lightlike submanifolds of an indefinite Sasakian manifold. $J$. Korean Soc. Math. Educ. Ser. B: Pure Appl. Math. 18 (2011), no. 1, 51-61.

11. _ : Half lightlike submanifolds of an indefinite Sasakian manifold. J. Korean Soc. Math. Educ. Ser. B: Pure Appl. Math. 18 (2011), no. 2, 173-183.

12. $\quad$ : Geometry of lightlike hypersurfaces of an indefinite cosymplectic manifold. Commun. Korean Math. Soc. 27 (2012), no. 1, 185-195.

13. $\quad$ S Special half lightlike submanifolds of an indefinite cosymplectic manifold. $J$. Function Spaces and Appl. Article ID 636242 (2012), 1-16.

14. Jin, D.H \& Lee, J.W.: Generic lightlike submanifolds of an indefinite cosymplectic manifold. Mathematical Problems in Engineering. Article ID 610986 (2011), 1-16.

15. Kim, S.K.: Lightlike Submanifolds of Indefinite Cosymplectic manifolds. Ph.D. Thesis, Ulsan University, Korea, 2007.

16. Kupeli, D.N.: Singular Semi-Riemannian Geometry. Mathematics and its Applications, Kluwer Acad. Publishers, Dordrecht, 1996.

Department of Mathematics, Dongguk University, Gyeongue 780-714, Republic of KoREA

Email address: jindh@dongguk.ac.kr 